\title{
Conjugate observation of sharp dynamical boundary in the inner magnetosphere by Cluster and DMSP spacecraft and ground network
}

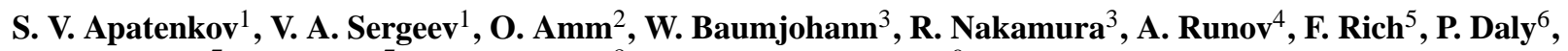 \\ A. Fazakerley ${ }^{7}$, I. Alexeev ${ }^{7}$, J. A. Sauvaud ${ }^{8}$, and Yu. Chotyaintsev ${ }^{9}$ \\ ${ }^{1}$ St. Petersburg State University, St. Petersburg, Russia \\ ${ }^{2}$ Finnish Meteorological Institute, Helsinki, Finland \\ ${ }^{3}$ Space Research Institute, Austrian Academy of Sciences, Graz, Austria \\ ${ }^{4}$ Institute of Geophysics and Planetary Physics, UCLA, Los Angeles, CA, USA \\ ${ }^{5}$ AFRL, Hanscom Air Force Base, MA01731, USA \\ ${ }^{6}$ Max Planck Institute for Solar System Research, Katlenburg-Lindau, Germany \\ ${ }^{7}$ Mullard Space Science Laboratory, University College London, UK \\ ${ }^{8}$ Centre d'Etude Spatiale des Rayonnements, Toulouse, France \\ ${ }^{9}$ Swedish Institute of Space Physics, Uppsala, Sweden
}

Received: 12 March 2008 - Revised: 15 July 2008 - Accepted: 12 August 2008 - Published: 17 September 2008

\begin{abstract}
We investigate an unusual sharp boundary separating two plasma populations (inner magnetospheric plasma with high fluxes of energetic particles and plasma sheet) observed by the Cluster quartet near its perigee on 16 December 2003. Cluster was in a pearl-on-string configuration at 05:00 MLT and mapped along magnetic field lines to $~ 8-$ $9 R_{E}$ in the equatorial plane. It was conjugate to the MIRACLE network and the DMSP F16 spacecraft passed close to Cluster footpoint. The properties of the sharp boundary, repeatedly crossed 7 times by five spacecraft during $\sim 10 \mathrm{~min}$, are: (1) upward FAC sheet at the boundary with $\sim 30 \mathrm{nA} / \mathrm{m}^{2}$ current density at Cluster and $\sim 2000 \mathrm{nA} / \mathrm{m}^{2}$ at DMSP; (2) the boundary had an embedded layered structure with different thickness scales, the electron population transition was at $\sim 20 \mathrm{~km}$ scale at Cluster ( $<7 \mathrm{~km}$ at DMSP), proton population had a scale $\sim 100 \mathrm{~km}$, while the FAC sheet thickness was estimated to be $\sim 500 \mathrm{~km}$ at Cluster ( $\sim 100 \mathrm{~km}$ at DMSP); (3) the boundary propagated in the earthward-eastward direction at $\sim 8 \mathrm{~km} / \mathrm{s}$ in situ (equatorward-eastward $\sim 0.8 \mathrm{~km} / \mathrm{s}$ in ionosphere), and then decelerated and/or stopped. We discuss the boundary formation by the collision of two different plasmas which may include dynamical three-dimensional fieldaligned current loops.
\end{abstract}

Correspondence to: S. V. Apatenkov (apatenkov@geo.phys.spbu.ru)
Keywords. Magnetospheric physics (Current systems; Energetic particles, trapped; Magnetopause, cusp, and boundary layers)

\section{Introduction}

The energetic particle population in the inner magnetosphere and the radiation belts, organized by particle drifts, is known to be rather stable except for energetic particle injection intervals and magnetic storm times, when the major magnetic reconfigurations occur and the transport of plasma and energy is strongly enhanced (e.g. Friedel et al., 2001).

Investigating the Cluster perigee passes during 2001-2006 we found several cases of sharp changes in the energetic particles flux which sometimes occur inside the radiation belt, at locations presumably mapped to equatorial distances of 6-10 $R_{E}$. Some of these flux changes (about one order of magnitude) consisted of successive sharp decreases and increases separated by $2-10 \mathrm{~min}$. To our knowledge this phenomenon has not been properly investigated in the literature before. In this paper we study in detail one of such events registered on 16 December 2003 by the Cluster spacecraft, which had $<1000 \mathrm{~km}$ separation.

The event of interest developed during moderate activity with $\mathrm{AE}$ index $\sim 200 \mathrm{nT}$ (i.e. under the modest convection),

Published by Copernicus Publications on behalf of the European Geosciences Union. 


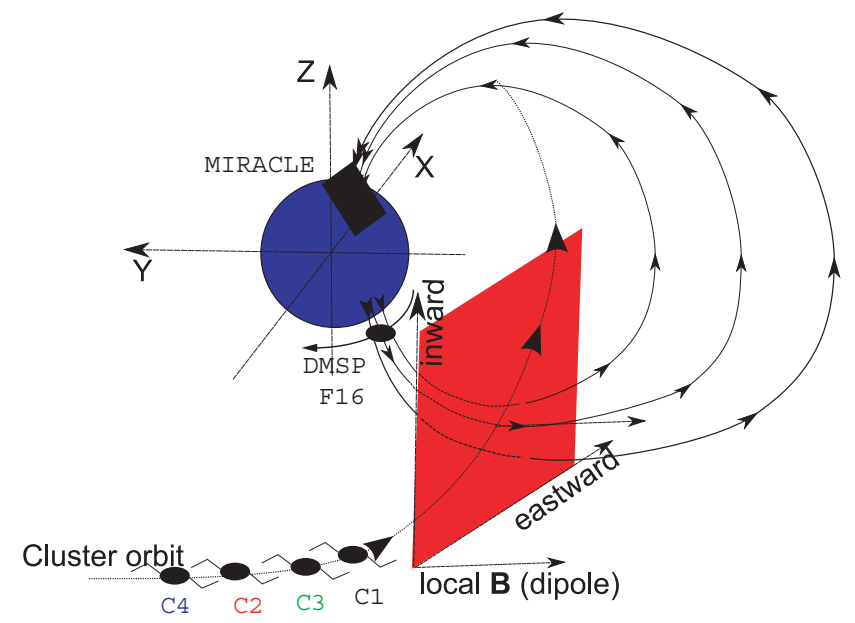

Fig. 1. Spacecraft location and the local reference frame chart. The orbit segments and directions for DMSP F16 and Cluster quartet in pearl-on-string are presented. The plane $\left(\boldsymbol{e}_{2} \boldsymbol{e}_{3}\right)$ orthogonal to local magnetic field is shown by red. Cluster and DMSP are at 05:00 MLT and conjugate to MIRACLE ground network.

when no concurrent energetic particle injections were observed at $6.6 R_{E}$. This makes the observed phenomena, the sharp changes in the energetic population, interesting and unusual by itself. The observed boundary has the thickness compared to ion gyroradius and the lifetime more than $10 \mathrm{~min}$. Taking into account that such a boundary is very important plasma physical object, potential site for different instabilities to occur, we investigate the plasma properties across this boundary, the boundary orientation, dynamics and the current systems. We shall finally discuss possible interpretations of such phenomena.

\section{Spacecraft configuration and instruments}

On 16 December 2003 02:15-02:30 UT Cluster approached its perigee $\left(4 R_{E}\right)$ at $\sim 05: 00$ MLT meridian, see Fig. 1 . The spacecraft were in pearl-on-string configuration following each other along their trajectory. The separation between the leading (C1) and trailing (C4) spacecraft was about $1000 \mathrm{~km}$. The spacecraft moved inward in terms of equatorial projection.

In the present study we use the following set of instruments. Cluster magnetic field with $1 \mathrm{~s}$ resolution and electric field with spin resolution ( $4 \mathrm{~s}$ ) are provided by FGM (Balogh et al., 2001) and EFW (Gustafsson et al., 2001) instruments respectively. Energetic electron and proton fluxes with spin resolution come from RAPID (Wilken et al., 2001). We also use auroral energy ion observations from CIS (Rème et al., 2001) (only Cluster 4 data were available for the event) and auroral energy electrons from the PEACE instrument (Johnstone et al., 1997). The set of DMSP instruments is described in Rich et al. (1985). Ground magnetic field near the Cluster ionospheric footpoint is provided by the dense array of MIRACLE/IMAGE magnetometers (Syrjäsuo et al., 1998).

\section{Observations}

The event of interest occurred during the prolonged interval (several hours) of high speed solar wind (about $650 \mathrm{~km} / \mathrm{s}$ ), the dynamic pressure was varying around $2 \mathrm{nPa}$. The interplanetary magnetic field had absolute value $4-5 \mathrm{nT}$ with $B_{Z}$ component varying in between -2 and $2 \mathrm{nT}$. Magnetic activity in the auroral zone was moderate, the $\mathrm{AL}$ and $\mathrm{AE}$ indices did not exceed $200 \mathrm{nT}$ in absolute value.

\subsection{Plasma observations at Cluster}

The sharp boundaries in question can be most easily recognized in the energetic particles (RAPID) observations, presented in Fig. 2a-c. At 02:16 UT Cluster has already entered the radiation belts and measured high fluxes, $\sim 20 \%$ of the peak values observed at $4 R_{E}$ perigee. Then at 02:19 UT Cluster 2, 3 and 4 spacecraft observed a sudden flux decrease of the energetic electrons $(E>50 \mathrm{keV})$ and protons $(E>27 \mathrm{keV})$ which was followed by a fast flux increase at 02:24 UT; the flux drop was more than one order of amplitude at $\mathrm{C} 2,3,4$. $\mathrm{C} 4$ observed the flux decrease first, then $\mathrm{C} 2$ and $\mathrm{C} 3$ did. The details of delays in the boundary observation time at three spacecrafts will be discussed below. No comparable boundaries are seen at the leading (inwardmost) Cluster 1 ; it observed only some small variations in the energetic electron fluxes during 02:19:30-02:21:00.

Two main features must be noted in these observations: (1) the transition from high to low fluxes and back was very fast, during 2-3 spins (8-12 s), and (2) the electrons at all RAPID energy channels, up to $344 \mathrm{keV}$, were affected by these changes simultaneously. We also notice that the boundaries in energetic protons are smoother than in electrons, the transition took 4-5 spins (16-20s).

The spectrograms of auroral energy plasmas, electrons below $24 \mathrm{keV}$ (PEACE) and protons below $30 \mathrm{keV}$ (CIS), are shown in Fig. 3. The boundaries in PEACE electrons were observed at the same time as in RAPID at C2, C3 and C4, Fig. 2a-c. The boundary passage here takes only $1-2$ spins (4-8 s). The sharp flux decrease (and the increase following in $5 \mathrm{~min}$ ) was observed at energies higher than $1-2 \mathrm{keV}$ and showed no dispersion in the whole energy range from $2 \mathrm{keV}$ to $344 \mathrm{keV}$ ( $>2$ orders of magnitude). In between these transitions (02:19-02:24) we observe not only the drop of flux at high energies, but the low energy $(0.1-2 \mathrm{keV})$ channels show the flux increases. In other words, the spacecraft suddenly moved into the different plasma population with a low temperature of electrons $(\sim 0.1-0.5 \mathrm{keV})$ as compared with higher temperature plasma $(\sim 2-3 \mathrm{keV})$ with pronounced high-energy tail in the energy spectra which was 
observed before and after the boundaries. We also notice that field-aligned beams of electrons $0.5-10 \mathrm{keV}$ in both directions can be seen at the boundaries. This feature is repeatable at all four spacecraft, however, only Cluster 4 data are shown in Fig. 3a where the field-aligned beams can be recognized in $1 . .14 \mathrm{keV}$ channels at $02: 18: 30$ and in $7.2 . .14 \mathrm{keV}$ channels at 02:24:02.

The ions show a similar behavior. Before and after the boundaries an ion population with $T \sim 5-6 \mathrm{keV}$ is observed, in between the boundaries, the flux at $E>10 \mathrm{keV}$ decreases and the high-energy population is substituted with colder plasma $(T \sim 2 \mathrm{keV})$. Field-aligned ion beams (ionospheric outflow) are clearly distinguished at the first boundary, Fig. 3c, at 02:18:30-02:19:00.

These plasma observations show that the spacecraft crossed twice a boundary separating two different plasma populations: (1) hot radiation belt-like plasma and (2) relatively cold, plasma sheet-like plasma. The field-aligned beams are observed both in electron and ion measurements at the boundary. Ions beams are ionospheric outflows, while electron beams come in both directions.

3.2 Cluster B- and E- field observations and boundary orientation

At the time of boundary crossings Cluster was moving in a strong magnetic field $\sim 400 \mathrm{nT}$, whereas the variations related to the plasma structure of interest were of several tens of nanotesla only. To facilitate the investigation we subtract the Earth's field contribution (IGRF) from the Cluster magnetic field data, $\Delta \boldsymbol{B}=\boldsymbol{B}_{\mathrm{Cluster}}-\boldsymbol{B}_{\mathrm{IGRF}}$ and rotate these field variation into B-aligned reference frame. Here the first axis $\boldsymbol{e}_{1}$ is along the local IGRF B-direction, we introduce $\boldsymbol{e}_{2}=-\left[\boldsymbol{e}_{1} \times \boldsymbol{R}\right] /\left|\boldsymbol{e}_{1} \times \boldsymbol{R}\right|$, where $\boldsymbol{R}$ is the radius vector, and $\boldsymbol{e}_{3}$ completes the 3 right-handed unit vectors. So $\boldsymbol{e}_{2}$ roughly corresponds to westward azimuthal direction and $\boldsymbol{e}_{3}-$ to the outward radial direction as shown in Fig. 1.

The magnetic field variations in B-aligned reference frame are shown in Fig. 2c-e. It is clearly seen that there were almost no variations in the $B_{1}$ (field-aligned or compressional) component as compared to the $B_{2}$ and $B_{3}$ components. Thus we believe that field-aligned currents are responsible for these magnetic variations. We also notice that the variations in the radial $\left(B_{3}\right)$ component are comparable with those in the azimuthal $\left(B_{2}\right)$ component. That means if the 1$\mathrm{d}$ sheet geometry is valid, the FAC sheet is tilted with respect to the local $\boldsymbol{e}_{1} \boldsymbol{e}_{2}$ plane.

To check if the FAC was a 1-D layer we draw the hodogram with $B_{2}$ versus $B_{3}$ for every spacecraft. The intervals with the largest field gradients are selected, marked by horizontal bars of corresponding colors in Fig. 2e, f. These intervals are about 2 min long, centered near 02:19UT for the first crossing and near 02:24 UT for the second one. The hodograms presented in Fig. 3a-c show the nearly-linear polarization of the magnetic variations for both crossings, the

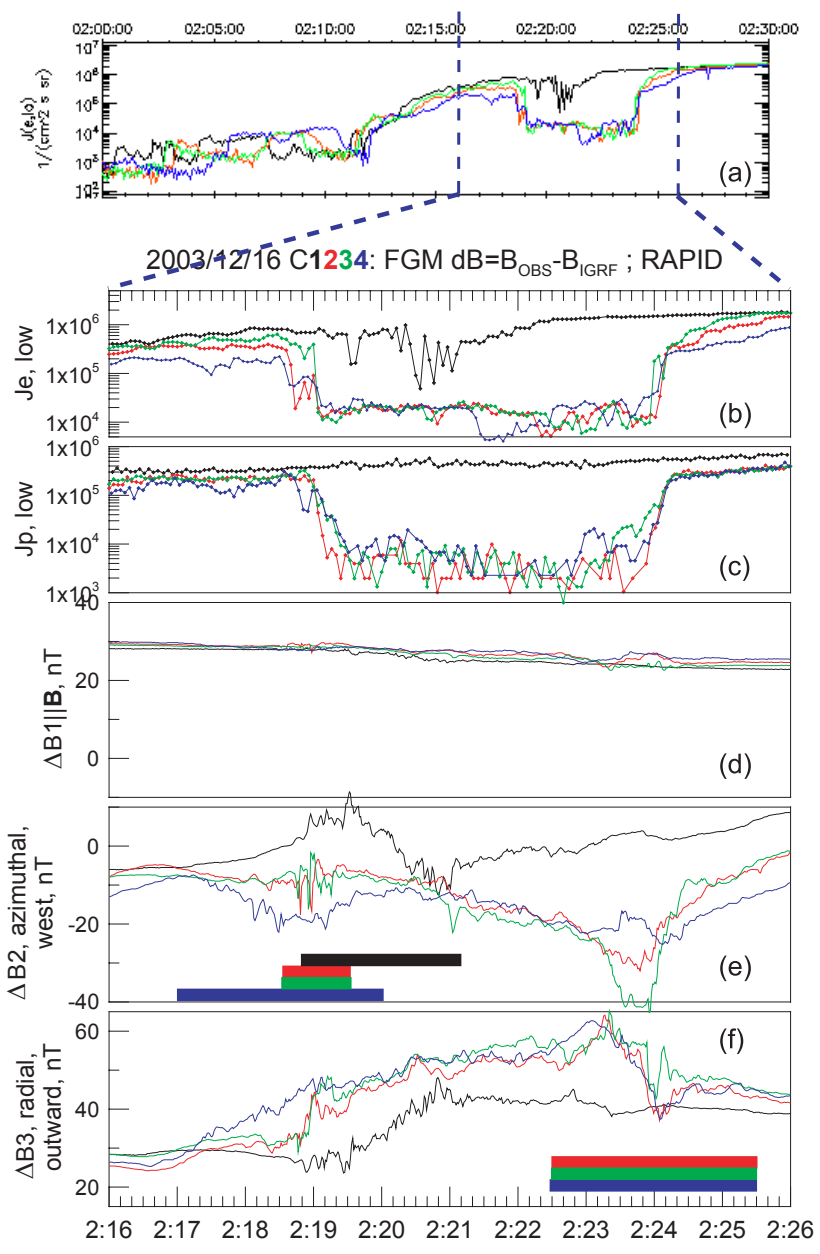

Fig. 2. (a-c) Energetic particle from Cluster RAPID, integral fluxes of electrons $(E>50 \mathrm{keV})$ and protons $(E>27 \mathrm{keV})$; $(\mathbf{d}-\mathbf{f})$ the magnetic variations in the local reference frame with subtracted IGRF. The color bars show the time intervals with the largest variations used to build the hodograms of $\Delta B_{2}$ versus $\Delta B_{3}$ components in Fig. 4. Black, red, green, blue correspond to Cluster 1, 2, 3, 4, respectively.

orientation of the both boundaries is similar, with the normal pointing in eastward-earthward direction.

The EFW double probe instrument provides the electric field measurements in the spin plane, which is close to GSE $\mathrm{XY}$ plane. Assuming the absence of parallel electric field at Cluster altitudes $(>30000 \mathrm{~km}$ from the ionosphere along the field line), i.e. $\boldsymbol{B} \cdot \boldsymbol{E}=0$, allows to obtain the third electric field component. (One should be careful using this procedure if magnetic field vector approaches the spin plane. We discard the points when the angle $\angle\left(B, X Y_{\mathrm{GSE}}\right)<5^{\circ}$.) The electric field is presented in Fig. 4 in the local-B reference frame. The largest variations peak-to-peak were registered at the boundaries, where they reached $100 \mathrm{mV} / \mathrm{m}$. 


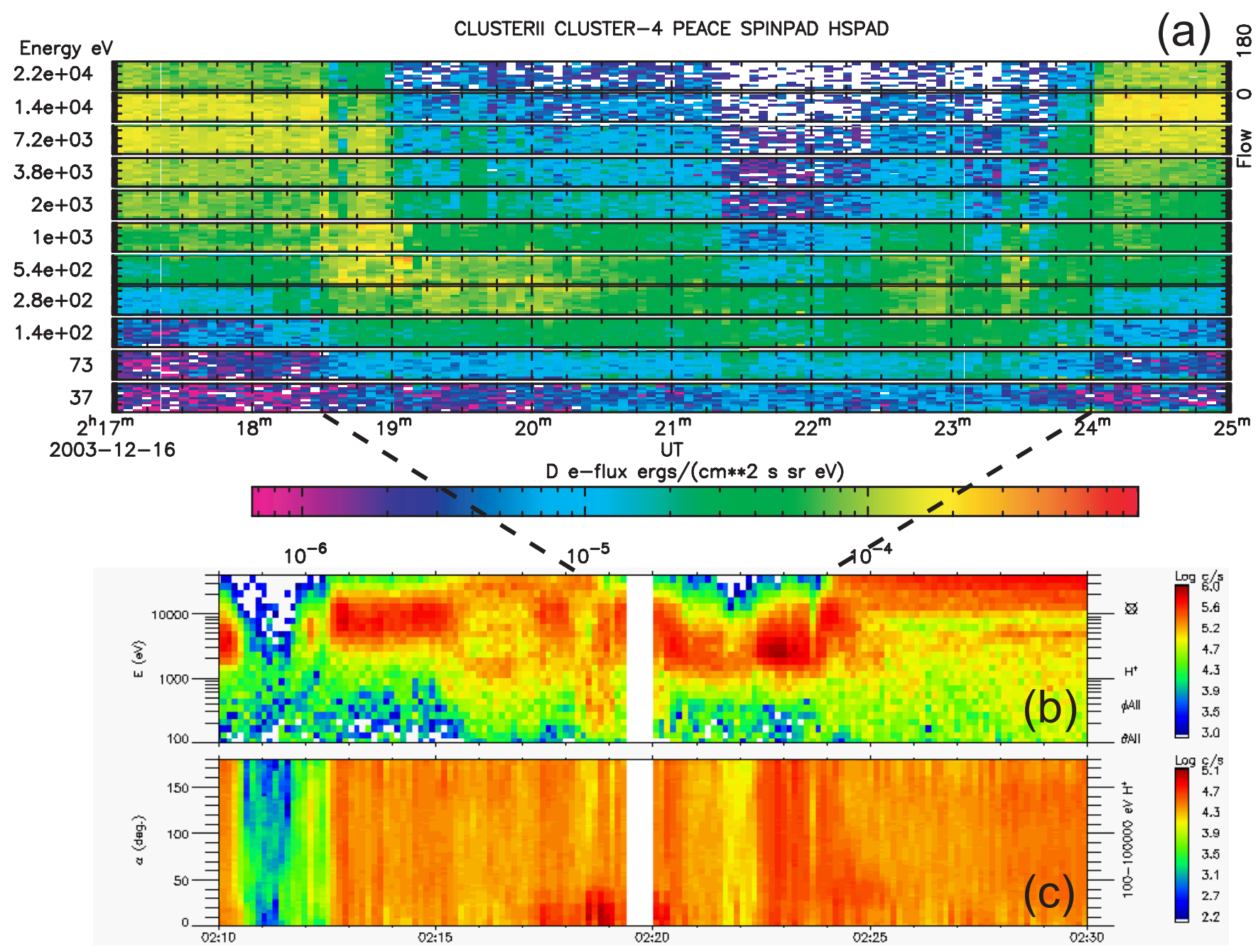

Fig. 3. Cluster 4 plasma observations. (a) PEACE spectrogram, every third energy channel is shown, the pitch-angle distributions are in the horizontal bars $\left(0^{\circ}-\right.$ bottom of the bar, $180^{\circ}-$ top) corresponding to different energies. (b) CIS/CODIF ion spectrogram and (c) $0.1-10 \mathrm{keV}$ ion pitch-angle distribution.

We draw the hodograms of $E_{2}$ versus $E_{3}$ components for the same time intervals as for the magnetic field (Fig. $4 \mathrm{~b}$, $\mathrm{c}, \mathrm{d}, \mathrm{e})$. Electric field variations are linearly polarized and their hodogram axis appeared to be almost perpendicular to the magnetic axis with the electric field vectors looking towards the FAC sheet center - that corresponds to a negatively charged layer.

Agreement between the magnetic and electric field hodograms together with a simple, nearly-linear polarization of the hodograms in most cases indicate that a sheet-like geometry is a reasonable assumption.

Taking into account the boundary orientation we can estimate the propagation speed. Using the starts of the energetic electron flux drops, 02:18:30 at $\mathrm{C} 4,02: 18: 40$ at $\mathrm{C} 2$ and 02:19:00 at $\mathrm{C} 3$, we deduce that the boundary passed approximately $250-400 \mathrm{~km}$ in $30 \mathrm{~s}$, it corresponds to $8-13 \mathrm{~km} / \mathrm{s}$ for the first crossing after subtracting the spacecraft motion which is $\sim 5 \mathrm{~km} / \mathrm{s}$ in the $\boldsymbol{e}_{2} \boldsymbol{e}_{3}$ plane. In between two boundaries we also notice some variations in RAPID electron flux at $\mathrm{C} 1$ during 02:19:20-02:21:10, Fig. 2b: it shows that the boundary touched $\mathrm{C} 1$ several times or was moving close to it. In the last case it should have had the speed $4-6 \mathrm{~km} / \mathrm{s}$. The second crossing was observed almost simultaneously at $\mathrm{C} 2$, $\mathrm{C} 3$ and $\mathrm{C} 4$. We suppose the boundary motion was considerably decelerated, almost stopped, and it was crossed by three moving spacecraft.

We estimate the field-aligned current density assuming a one-dimentional layer $j=\frac{\Delta B}{\mu_{0} \Delta d}$ where $B$ and $d$ correspond to a jump of tangential magnetic field and the layer thickness, respectively. Taking magnetic field in $\mathrm{nT}$ and distance in $1000 \mathrm{~km}$ we get $0.796 \cdot \Delta B / \Delta d$ for current density in $\mathrm{nA} / \mathrm{m}^{2}$. The duration of the magnetic variations (20-30 nT 
in magnitude) at the first boundary is about $50-200 \mathrm{~s}$, corresponding to a FAC thickness of $250-700 \mathrm{~km}$. So the current density is estimated to be roughly $20-90 \mathrm{nA} / \mathrm{m}^{2}$. The differences in the magnetic field between the spacecraft located at the opposite sides of the boundary correspond to the upward FAC direction during the both crossings.

Two successive boundary crossings at Cluster showed the similar properties, in both cases we observe the upward FAC sheet and the similar orientation. We suppose this is the same boundary which was crossed twice due to the decreasing propagation speed. The boundary propagation revealed from all available observations is further discussed in the Sect. 3.5.

\subsection{Magnetic field configuration}

To interpret joinly the magnetospheric observations with the low-altitude observations we have to provide the mapping along the field lines using an appropriate model of the magnetospheric magnetic field. For this purpose we use Tsyganenko 89 model (Tsyganenko, 1989) parametrized by $K_{p}$ index. We select the $K_{p}$ parameter which reproduces best the magnetospheric magnetic field observed at Cluster. Cluster magnetic field observations during 02:10-02:30 UT are in a good correspondence with $\mathrm{T} 89$ with $K_{p}=4$ (not shown). According to this model Cluster were mapped to $8-9 R_{E}$ at the equatorial plane.

\subsection{Low-altitude DMSP observations}

During the time of interest DMSP F16 was moving poleward along the $\sim 05: 00$ MLT meridian in the proximity of Cluster ionospheric footpoint. It observed high fluxes of electrons with energies 1-10 keV till 03:27:10 UT, Fig. 5c. Then suddenly, during less than $1 \mathrm{~s}$, at $\sim-66^{\circ}$ magnetic latitude, the population of $E>5 \mathrm{keV}$ electrons disappeared and electron average energy changed from $10 \mathrm{keV}$ to $1 \mathrm{keV}$. Such a fast transition reveals the boundary thickness of $<7 \mathrm{~km}$ at DMSP altitude $(850 \mathrm{~km})$, here high spacecraft speed $\sim 7 \mathrm{~km} / \mathrm{s}$ allows to consider the boundary motion negligibly slow.

To our knowledge, such a boundary has not been specially discussed in the literature. It does not necessarily mean this boundary was rarely observed at DMSP, we believe it has not been paid attention much. One can notice some similarity with DMSP observations during auroral streamer crossings presented in Sergeev et al. (2004). However, the boundary in our observations is sharper and the plasma temperature at the equatorial side is higher.

This boundary is detected in the magnetic field and ion drift as well. Figure 5b shows the magnetic variations recorded by DMSP/SSM instrument in the horizontal plane, here Y-DMSP direction is along spacecraft velocity (roughly poleward), Z-DMSP is normal to velocity (roughly westward). The largest negative variation was observed in the $B_{Z}$ component, across the track, with the variation sign corresponding to upward FAC. The one-dimensional geometry
Cluster EFW - 2003/12/16

observe Ex,Ey GSE; use $\left(E^{*} B\right)=0$; rotate to local-B frame
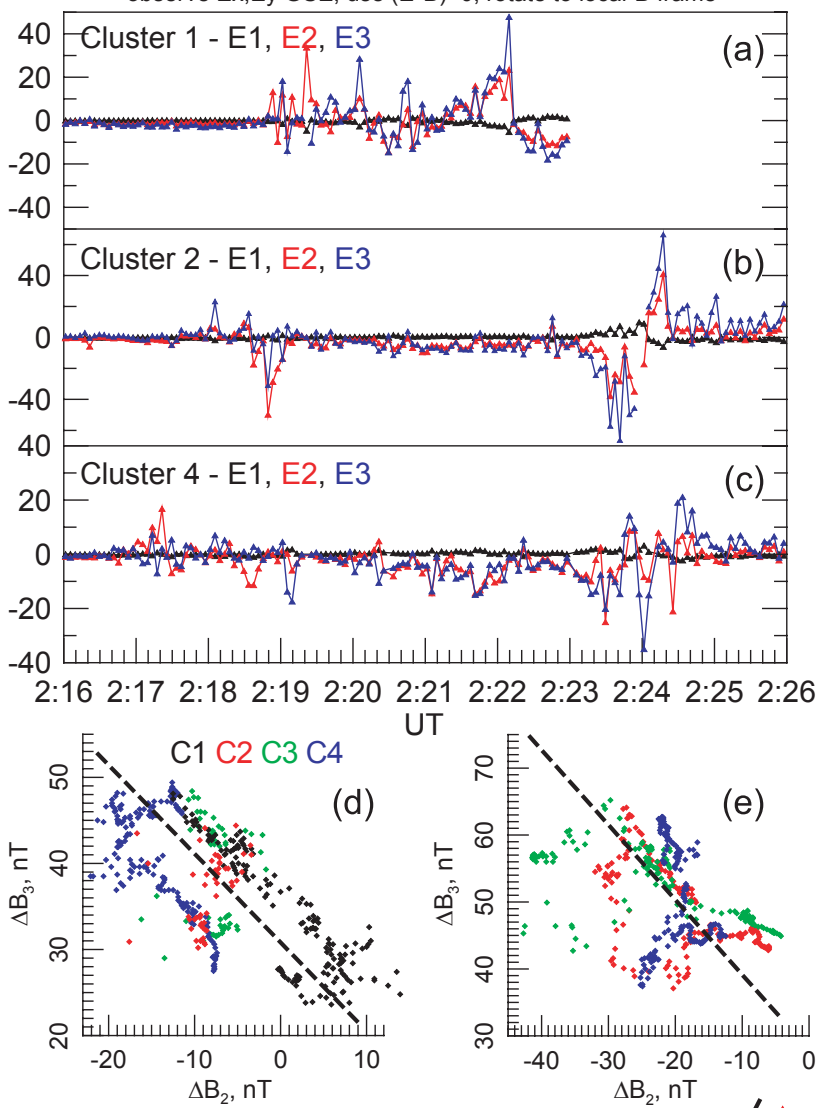

UT
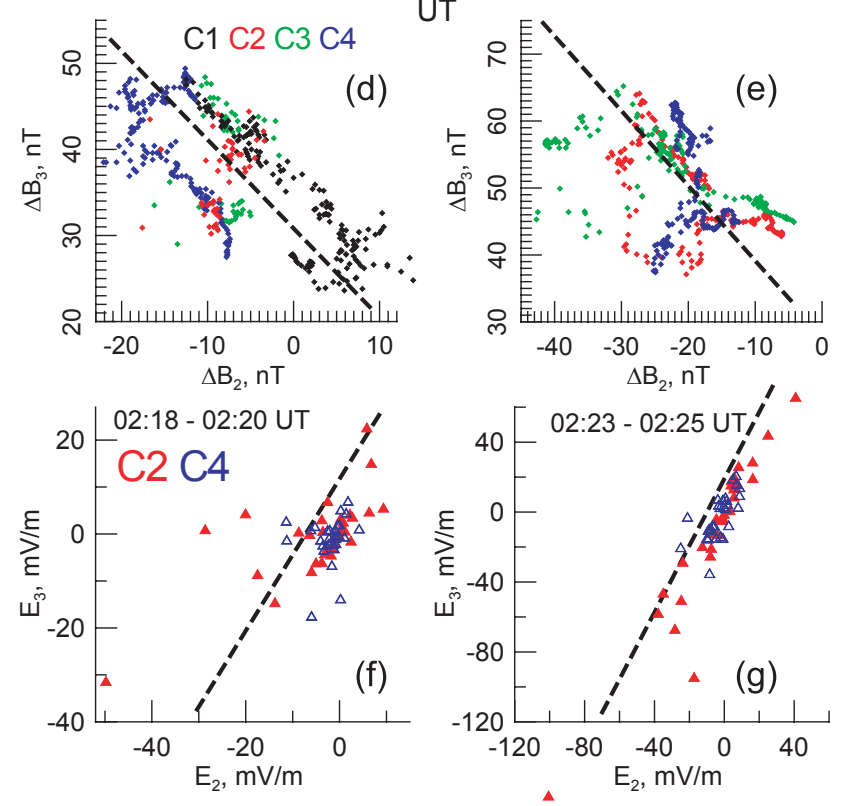

Fig. 4. Electric field variations from Cluster EFW (a-c). Hodograms of B-field, $\Delta B_{2}$ versus $\Delta B_{3}$ components (d-e) and Efield, $E_{2}$ versus $E_{3}$ components (f-g). Hodograms show nearly linear polarization that confirms one-dimentionality of the boundary.

and the orientation are clearly identified from the hodogram in the same manner like we did at Cluster, Fig. 5f. Fieldaligned current sheet thickness is approximately $100 \mathrm{~km}$, current density is $\sim 2000 \mathrm{nA} / \mathrm{m}^{2}$. Schematically this boundary shown in Fig. 6 crossing the DMSP trajectory, the FAC sheet has $\sim 10^{\circ}$ angle with the geomagnetic latitude.

Two components, vertical (upward) and horizontal ( eastward), of the ion drift velocity from DMSP/SSIES instrument are presented in Fig. 5e. At around 02:28:15 the 


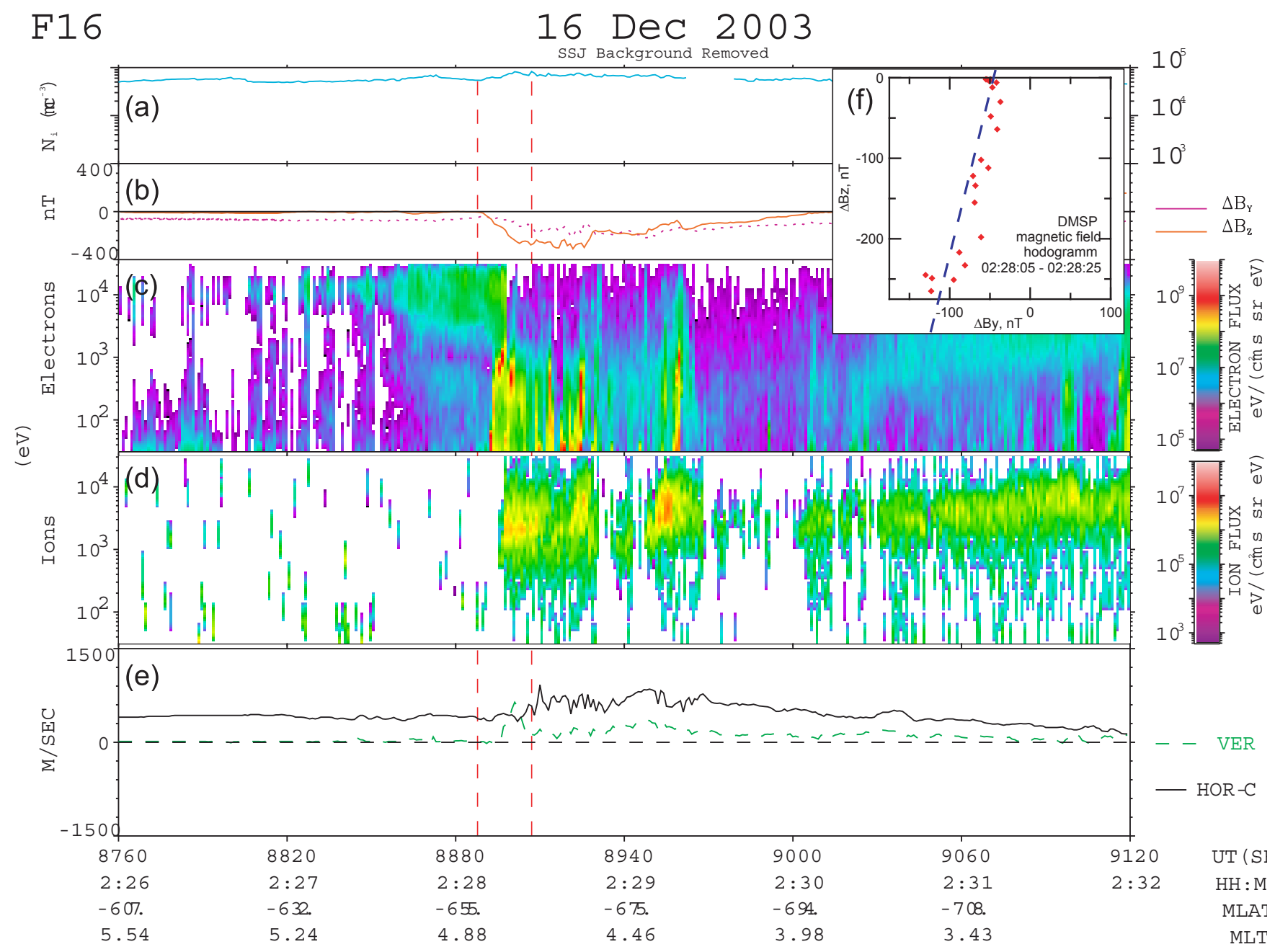

Fig. 5. DMSP F16 observations, (a) plasma density, (b) magnetic variations, (c-d) electron and ion energy flux spectrogram, (e) ion drift velocities, (f) hodogram of magnetic field horizontal components. Two red vertical dashed lines bracket the magnetic field variations related to the boundary crossing.

velocity shear is observed. The drift velocity equatorward of the boundary is $\sim 400 \mathrm{~km} / \mathrm{s}$, it jumps to $\sim 700 \mathrm{~km} / \mathrm{s}$ behind the boundary. This shear correspond to the electric field converging to the boundary, which agrees with the upward FAC sheet.

3.5 Ground observations at IMAGE network - boundary dynamics

The Cluster footpoint in the northern ionosphere passes above the IMAGE magnetometer network. The auroral magnetometers recorded weak variations of 10-20 nT in all three components during the event of interest, 02:10-02:30 UT. These variations are rather weak on the background of a recovering negative bay in $B_{X}$ component with 150-200 nT amplitude related to a westward electrojet, Fig. 7 a.
Using 2-D upward continuation method (Amm and Viljanen, 1999) we studied the 2-D pattern of the equivalent ionospheric currents and its dynamics during the period of interest. Figure $7 \mathrm{~b}$ shows the vertical component of the curl of equivalent ionospheric currents distribution, in case of uniform ionospheric conductance this quantity characterizes the field-aligned currents. The two areas, with upward FACs at the northern part and downward FACs at the southern part, are seen in Fig. 7, it corresponds to the westward electrojet. To investigate the dynamics of the additional localized current systems which are an order weaker than the electrojet is and therefor cannot be easily distinguished from the large scale current system we follow the dynamics of some distinct feature - the line where $\operatorname{curl}\left(\boldsymbol{J}_{E Q}\right)=0$.

This line roughly corresponds to the center of westward electrojet. It shows significant motion in equatorward 
direction slightly changing its shape. Its latitudinal behavior at $22^{\circ}$ longitude is presented in Fig. 8 (magenta line). Mapped trajectories of Cluster spacecraft and the boundaries observed in space are also plotted for comparison. $\operatorname{Curl}\left(\boldsymbol{J}_{E Q}\right)=0(\sim$ electrojet center $)$ starts to move equatorward at 02:17-02:18 UT, the motions continues till 02:22:30 UT, and after boundary stops and "fluctuates" at around the same location.

The boundaries between two different plasma populations observed at four Cluster spacecraft and then at low-altitude DMSP were mapped to the ionosphere as a function of time, they are marked by stars in Fig. 8. Cluster and DMSP F16 trajectories are also presented. We may discuss only normal to the boundary velocity component that is directed equatorward and has some slight eastward component. For the simplicity only the north-south magnetic coordinates of the spacecraft and boundary are presented in Fig. 8. Fast boundary motion at $\sim 0.8 \mathrm{~km} / \mathrm{s}$ speed at Cluster spacecraft around $02: 19$ UT changes with slower motion of $\sim 0.1-0.2 \mathrm{~km} / \mathrm{s}$ afterwards.

The time interval of fast boundary motion nicely agrees with the first boundary crossings at Cluster (Fig. 8). The second set of crossings at Cluster occurs after the stop of the boundary in the ionosphere. So in overall the dynamics of the boundary at Cluster and that in the ionosphere is similar although there is some difference in propagation speeds.

\section{Discussion and conclusions}

In the present study we investigate the distinct dynamical plasma boundary observed simultaneously by Cluster, DMSP, and the IMAGE magnetometer array in the morning sector ( $\sim 55: 00 \mathrm{MLT})$ of the inner magnetosphere at $8-9 R_{E}$ radial distance in the equatorial plane (when mapped according to T89 magnetospheric model with $K_{p}=4$ ). The boundary properties are the following:

1. The boundary separates two plasma populations. Earthward (equatorward) of the boundary high temperatures were observed: $T_{e} \sim 2-3 \mathrm{keV}, T_{i} 5-6 \mathrm{keV}$ (innermagnetosphere population). On the tailward side from the boundary: $T_{e} \sim 0.1-0.5 \mathrm{keV}, T_{i} \sim 2 \mathrm{keV}$ (plasma sheet-like population).

2. The upward field-aligned current layer was found at the boundary. The current density was $\sim 30 \mathrm{nA} / \mathrm{m}^{2}$ at Cluster and $\sim 2000 \mathrm{nA} / \mathrm{m}^{2}$ at DMSP, the current layer thickness values were $200-700 \mathrm{~km}$ and $100 \mathrm{~km}$, respectively. The values consistent with mapping scale factor between Cluster and DMSP that is about $\sqrt{50000 \mathrm{nT} / 400 \mathrm{nT}} \approx 11$.

3. The boundary at Cluster had the normal pointing in eastward-earthward direction. At low-altitude (DMSP) the FAC layer has $10-15^{\circ}$ angle with the geomagnetic

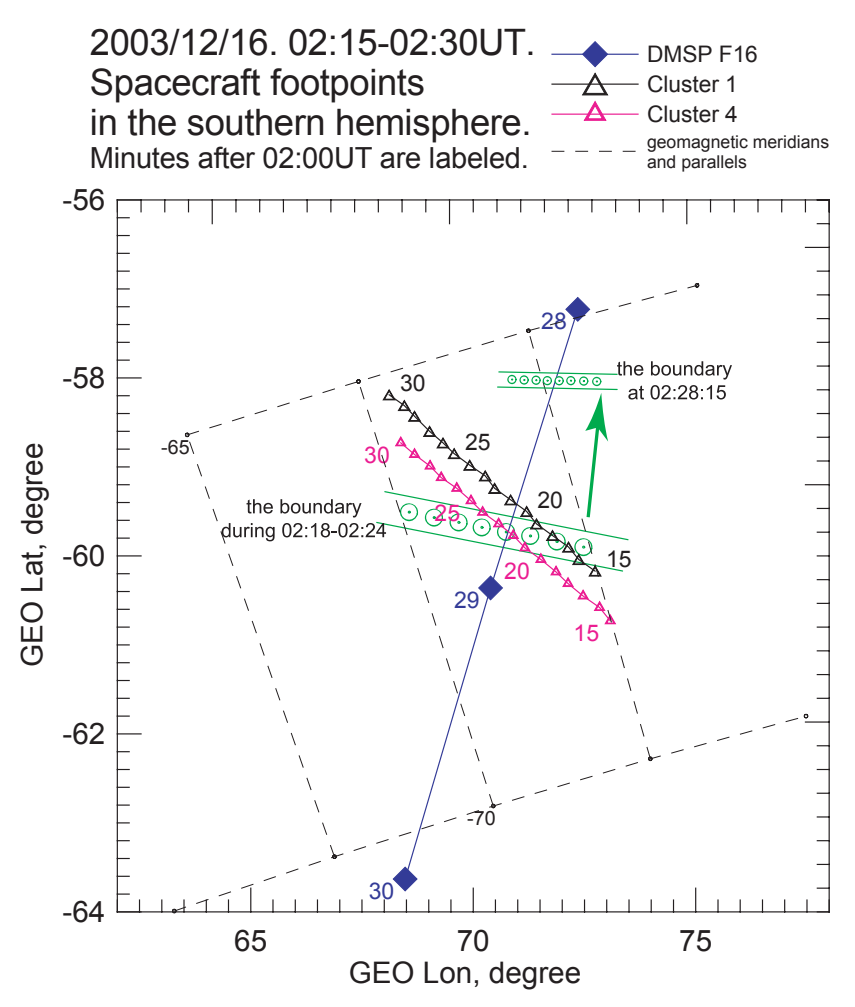

Fig. 6. Spacecraft footpoints at the southern ionosphere are shown by black, magenta (Cluster 1,4) and blue (DMSP F16) lines. Time is marked as minutes after 02:00 UT. Dashed lines show the grid of geomagnetic coordinates. The locations of the boundary(ies) with upward current are schematically presented by green.

parallel, the direction of rotation corresponds to the one found at Cluster.

4. The boundary moved earthward-dawnward with deceleration. The boundary velocity in the ionosphere decreased from $0.8 \mathrm{~km} / \mathrm{s}$ to $0.1 \mathrm{~km} / \mathrm{s}$, and the motion was in the equatorward-eastward direction. Equatorial (mapped) velocity was $\sim 60 \mathrm{~km} / \mathrm{s}$, it decelerated to almost zero during $10 \mathrm{~min}$.

5. As observed at Cluster, the boundary has a layered structure. The sharpest transition was observed in the electron parameters over $\sim 20 \mathrm{~km}$. The boundary in protons is about $100 \mathrm{~km}$ thick, i.e. comparable with a $10 \mathrm{keV}$ proton gyroradius. These sharp flux transitions are embedded into the FAC layer with 200$700 \mathrm{~km}$ thickness. The sketch of the boundary is shown in Fig. 9. At DMSP $(850 \mathrm{~km}$ altitude $)$ - the electron boundary had less than $7 \mathrm{~km}$ thickness and it was inside the FAC layer $\sim 100 \mathrm{~km}$ thick.

6. Two subsequent crossings of propagating boundary were observed at Cluster at $\sim 02: 18: 30$ and $\sim 02: 24: 00$. 


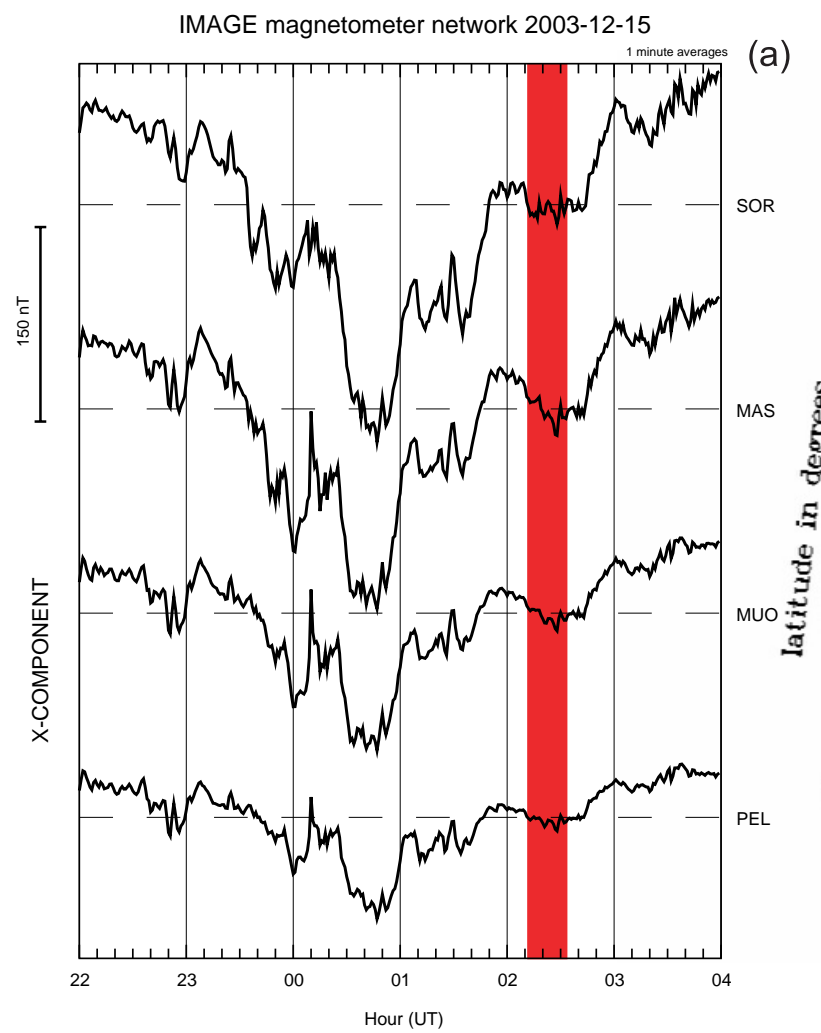

(b)

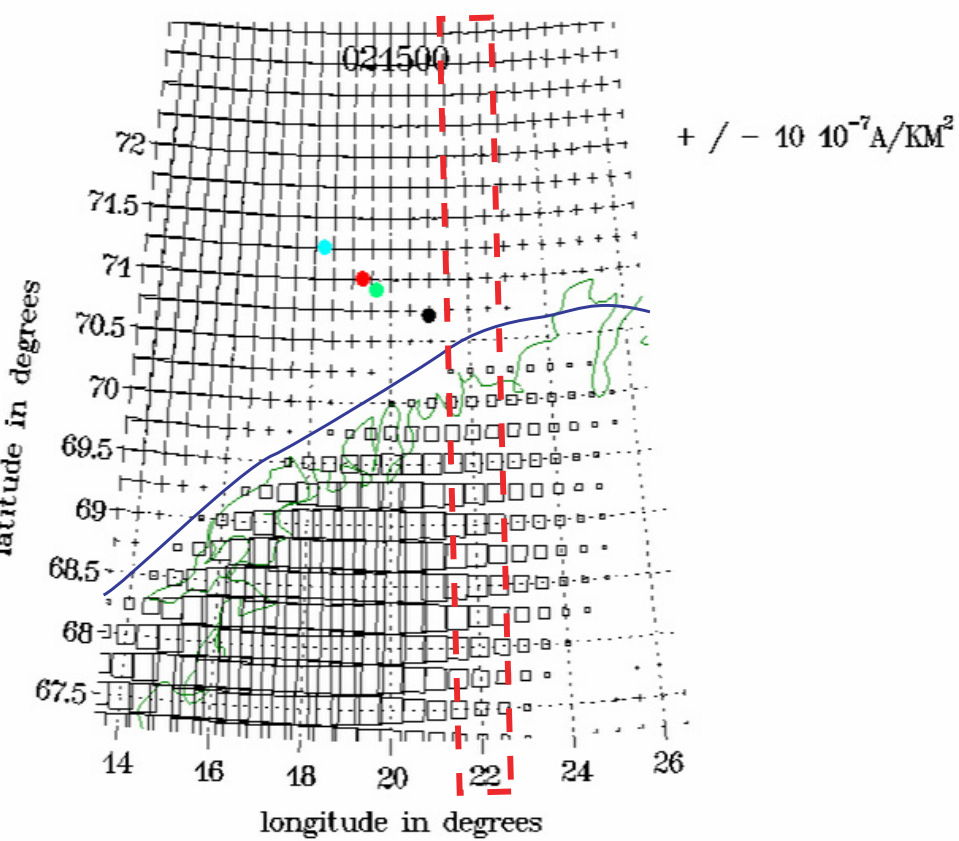

Fig. 7. (a) The X-component of magnetic variations from 5 IMAGE magnetometers, weak variations of interest at the background of the westward electrojet-related negative bay are marked by red bar. (b) Curl of ionospheric equivalent currents (after 2-D upward continuation), squares corresponds to upward FACs, crosses to downward, the $\operatorname{curl}\left(J_{E Q}\right)=0$ line related to the center of electrojet is shown by blue line. Red vertical dashed lines show the meridian used to extract latitude versus time bevavior in Fig. 8.

Then the boundary is detected as very distinct (not decaying) structure at DMSP at 02:28:10. So the lifetime of this plasma boundary is longer than $10 \mathrm{~min}$.

Similar properties (orientation, velocities, temperatures, FACs) were obtained in the analysis of one more event 27 December 2003, 23:50-23:59 UT when Cluster observed similar energetic particle flux variations. In that case Cluster was at $\sim 04: 00$ MLT and mapped to $\sim 8 R_{E}$ although there were neither ground nor low-altitude observational support.

Recently, in Sergeev et al. (2003), a sharp boundary with similar properties (thickness compared to ion gyroradius, no energy dispersion, electric field variations, plasma pressure change) was reported. However, some differences exist: the boundary was embedded in downward FAC sheet (rather that in upward FAC as in our case) and no boundary dynamics was seen in the observations.

Although we have no final interpretation for the phenomenon observed one may identify a number of important facts to be including when searching for its explanation.

The first question is - what creates this sharp boundary? We believe such sharp transition between two different populations can only be formed dynamically, as a result of col- lision of two different plasmas. The inferred earthwardeastward propagation supports this expectation. Particularly, this can happen during the injection of relatively cold tail plasma into the inner magnetosphere. Although known for decades, this phenomenon is scarcely investigated, especially as concerns the physics of its concluding stage. Bursty Bulk Flow (BBF, e.g. Angelopoulos et al., 1992) propagation earthward and eastward (around the strong-B region) or some BBF remnants (somehow transformed in the inner magnetosphere, e.g. Sergeev et al., 2000; Takada et al., 2006) can be considered as candidates for these inward intruding structures. The life time exceeding $10 \mathrm{~min}$ is consistent with a long lifetime of BBF optical remnants in the equatorward oval observed by the UVI imagers (Sergeev et al., 2005). The orientation of plasma boundaries at some angle with respect to the magnetic latitude, like shown in Fig. 8, is also consistent with the BBF hypothesis and observations: a few examples of auroral streamers in the morning sector with the corresponding orientation were demonstrated by Sergeev et al. (2004). According to the latter paper the upward FAC is a part of streamer structure located at its dusk side. If so the observed boundaries could be at the flanks of the $\mathrm{BBF} /$ streamer 


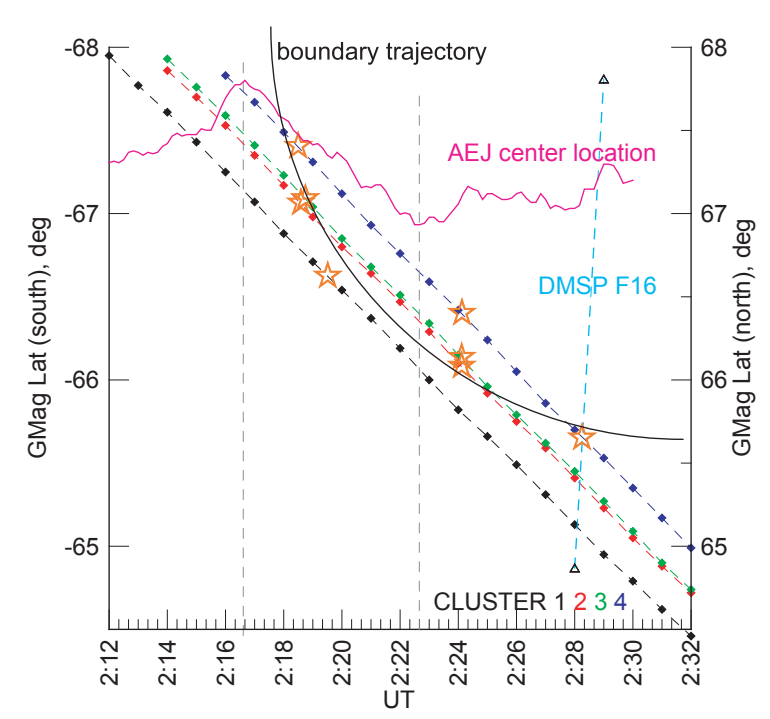

Fig. 8. Revealed boundary trajectory in magnetic latitude versus time coordinates. The locations of the Cluster (black, red, green, blue) and DMSP (light blue) spacecraft and the dynamics of the electrojet center (magenta) at $20^{\circ} \mathrm{E}$ longitude are also shown. Stars show the time when the boundary was detected by spacecraft. Vertical dashed lines bracket the time interval of suggested intense boundary motion.

structure which are crossed due to its motion, or due to overall change of the field-line shape.

An important aspect is however an off-equatorial position of the Cluster during observations of these transitions, which makes the interpretation more complicated. In this case the observed dynamics may also reflect the relative change in the shape of magnetic field-lines connected to two equatorial plasma sources, in addition to the actual relative motions of these sources. The field-aligned currents could be the important players in these configurational changes.

Another important question is - what prevents the radiation belt-like energetic particles from penetrating through the long-living boundary which is tilted at large angle with respect to the azimuthal direction, i.e. to the direction of nominal magnetic drift of the energetic particles? The only way can be if the magnetic configuration is changed locally in such a way, that the drift trajectory lines (e.g. $B=$ const for $90^{\circ}$ pitch angle particles) follow along this boundary. The relatively intense and meso-scale 3-dimensional current system, including the field-aligned currents, should be involved. A possible current system is a pair of separated azimuthally FAC sheets in opposite directions, the upward FAC is at the eastern side (in our case we observe it at the boundary) and the downward FAC is at the western side. In this configuration the equatorial magnetic field would decrease in between the currents and the trajectories of energetic particles would shift (be pushed) earthward in the local azimuthal sector. We do not specify the closure of these currents.

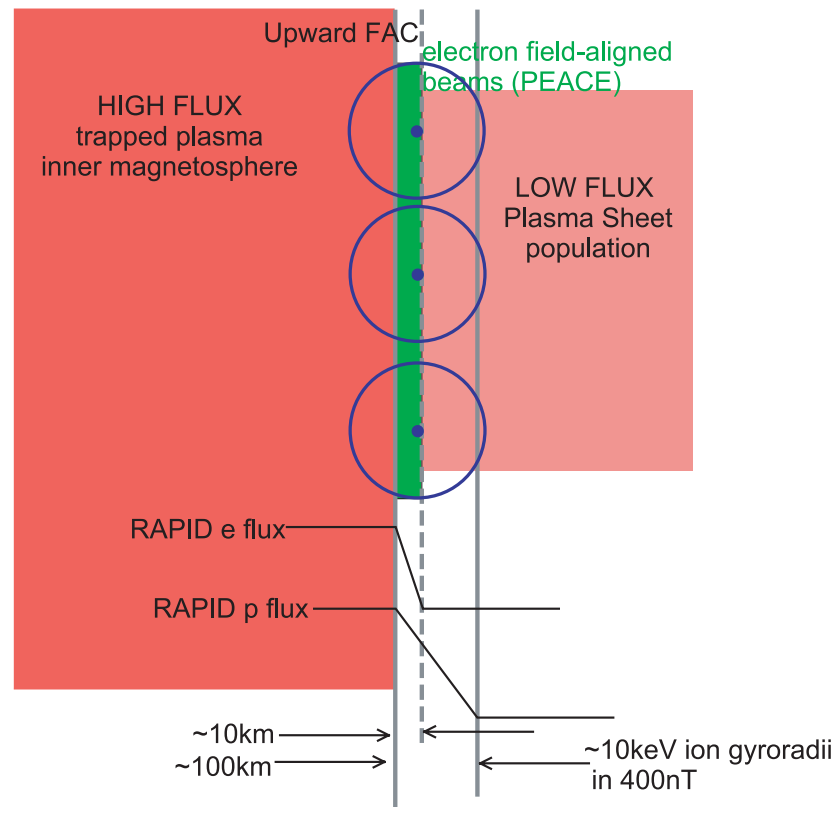

Fig. 9. Sketch of the boundary structure separating two plasma populations deduced from the observed parameters.

Some speculations regarding the phenomena providing such FAC pairs and their possible periodic spatial occurrence can be done. It is known that auroral omega bands usually appear in the morning LT sector as series of similar bright auroral tongues (or spots) and propagate eastward with velocities $0.1-2 \mathrm{~km} / \mathrm{s}$, in some cases the omega bands are formed from the equatorward propagating auroral streamers (Henderson et al., 2002; Sergeev et al., 2005). Each bright form has the current system containing of the upward - downward FAC pair connected via the ionosphere (Untiedt and Baumjohann, 1993; Amm et al., 2005). So potentially the omega bands may produce the required field-aligned currents and specific local magnetic configurations changes. As well the propagation direction and velocity fits those observed during the event of interest.

To conclude, we presented very clear examples showing that very narrow (of a few gyroradius scale) and electrically polarized plasma boundaries separating plasma sheet and hot innermagnetospheric populations can be observed for $>10 \mathrm{~min}$ inside the outer radiation belt. No theoretical model known to us is ready to explain the long-living thin plasma boundaries propagating in the inner magnetosphere. More investigations are required to identify the occurrence conditions, optical manifestations and origin to understand this important physical phenomenon, the sharp dynamical plasma boundaries.

Acknowledgements. Work by SA and VS was supported by INTAS grant 05-109-4496, by Russian Ministry of Education grants RNP.2.2.2.2.2190 and RNP.2.1.1.4166. Preliminary AE index was 
provided by Kyoto WDC-C data base. SA thanks Space Research Institute of Austrian Academy of Sciences and Finnish Meteorological Institute for support during the stay in Graz and Helsinki correspondingly.

Topical Editor I. A. Daglis thanks two anonymous referees for their help in evaluating this paper.

\section{References}

Amm, O. and Viljanen, A.: Ionospheric disturbance magnetic field continuation from the ground to the ionosphere using spherical elementary current systems, Earth, Planets Space, 51, 431-440, 1999.

Amm, O., Aksnes, A., Stadsnes, J., Ostgaard, N., Vondrak, R. R., Germany, G. A., Lu, G., and Viljanen, A.: Mesoscale ionospheric electrodynamics of omega bands determined from ground-based electromagnetic and satellite optical observations, Ann. Geophys., 23, 325-342, 2005,

http://www.ann-geophys.net/23/325/2005/.

Angelopoulos, V., Baumjohann, W., Kennel, C. F., Coroniti, F. V., et al.: Bursty bulk flows in the inner central plasma sheet, J. Geophys. Res., 97, 4027-4039, 1992.

Balogh, A., Carr, C. M., Acuna, M. H., et al.: The Cluster magnetic field investigations: overview of in-flight performance and initial results, Ann. Geophys., 19, 1207-1217, 2001, http://www.ann-geophys.net/19/1207/2001/.

Friedel, R. H. W., Korth, H., Henderson, M. G., Thomsen, M. F., and Scudder, J. D.: Plasma sheet access to the inner magnetosphere, J. Geophys. Res., 106(A4), 5845-5858, 2001.

Gustafsson, G., Andre, M., Carozzi, T., et al.: First results of electric field and density observations by Cluster EFW based on initial months of operation, Ann. Geophys., 19, 1219-1240, 2001, http://www.ann-geophys.net/19/1219/2001/.

Henderson, M. G.,Kepko, L., Spence, H. E., Connors, M., Sigwarth, J. B., Frank, L. A., Singer H. J., and Yumoto, K.: The Evolution of North-South Aligned Auroral Forms into Auroral Torch Structures: The Generation of Omega Bands and Ps6 Pulsations via Flow Bursts, Proc. Sixth International Conference on Substorms, Seattle, 25-29 March 2002, edited by: Winglee, R. M., 169-174, University of Washington press, 2002.

Johnstone, A. D., Alsop, C., Burge, S., et al.: Peace: a Plasma Electron and Current Experiment, Space Sci. Rev., 79, 351-398, 1997.

Rème, H., Aoustin, C., Bosqued, J. M., et al.: First multispacecraft ion measurements in and near the Earth's magnetosphere with the identical Cluster ion spectrometry (CIS) experiment, Ann. Geophys., 19, 1303-1354, 2001, http://www.ann-geophys.net/19/1303/2001/.
Rich, F. J., Hardy, D. A., and Gussenhoven, M. S.: Enhanced ionosphere-magnetosphere data from the DMSP satellites, EOS Trans. AGU, 66, 513, 1985.

Sergeev, V. A., Sauvaud, J.-A., Popescu, D., Kovrazhkin, R. A., Liou, K., Newell, P. T., Brittnacher, M., Parks, G., Nakamura, R., Mukai, T., and Reeves, G. D.: Multiple-spacecraft observation of a narrow transient plasma jet in the Earths plasma sheet, Geophys. Res. Lett., 27(6), 851-854, doi:10.1029/1999GL010729, 2000.

Sergeev, V. A., Sauvaud, J.-A., Reme, H., Balogh, A., Daly, P., Zong, Q.-G., Angelopoulos, V., Andre, M., and Vaivads, A.: Sharp boundary between the inner magnetosphere and active outer plasma sheet, Geophys. Res. Lett., 30(15), 1799, doi:10.1029/2003GL017095, 2003.

Sergeev, V. A., Liou, K, Newell, P. T., Ohtani, S.-I., Hairston, M. R., and Rich, F.: Auroral streamers: characteristics of associated precipitation, convection and field-aligned currents, Ann. Geophys., 22, 537-548, 2004, http://www.ann-geophys.net/22/537/2004/.

Sergeev, V. A., Yahnin, D. A., Liou, K., Thomsen, M. F., and Reeves, G. D.: Narrow Plasma Streams as a candidate to populate the inner magnetosphere, Geophysical Monograph Series, p.155, 2005.

Syrjäsuo, M., Pulkkinen, T. I., Pellinen, R. J., Janhunen, P., Kauristie, K., Viljanen, A., Opgenoorth, H. J., Karlsson, P., Wallman, S., Eglitis, P., Amm, O., Nielsen, E., and Thomas, C.: Observations of substorm electrodynamics using the MIRACLE network, Proc. Fourth International Conference on Substorms(ICS-4), Lake Hamana, Japan, 9-13 March 1998.

Takada, T., Nakamura, R., Baumjohann, W., Asano, Y., Volwerk, M., Zhang, T. L., Klecker, B., Rème, H., Lucek, E. A., and Carr, C.: Do BBFs contribute to inner magnetosphere dipolarizations: Concurrent Cluster and Double Star observations, Geophys. Res. Lett., 32, L21109, doi:10.1029/2006GL027440, 2006.

Tsyganenko, N. A.: A magnetospheric magnetic field with a warped tail current sheet, Planet. Space Sci., 37, 5-20, 1989.

Untiedt, J. and Baumjohann, W.: Studies of polar current systems using the IMS Scandinavian Magnetometer Array. Space Sci. Rev., 63, 245-390, 1993.

Wilken, B., Daly, P. W., Mall, U., et al.: First results from the RAPID imaging energetic particle spectrometer on board Cluster, Ann. Geophys., 19, 1355-1366, 2001, http://www.ann-geophys.net/19/1355/2001/. 